Classification

Physics Abstracts

$4265 \mathrm{~J}-32.80 \mathrm{P}$

\title{
Optical Pumping of Metastable Helium Atoms in Polarized Electron Sources
}

\author{
I. Brissaud \\ Institut de Physique Nucléaire, 91406 Orsay Cedex, France \\ (Received 1 June 1994, revised 12 December 1994, accepted 22 December 1994)
}

\begin{abstract}
In this paper we present a comparison between the Orsay and Rice University polarized electron sources. Different explanations of the polarization degradation are discussed for the two cases. For the Rice source radiation, trapping can account for the observed reduction in polarization because of the high absorption cross sections associated with the $\mathrm{D}_{1}$ transition Some improvements are proposed that might increase the electron polarization.
\end{abstract}

\section{Introduction}

Ten years ago Walters et al. [1] designed a polarized electron source using a helium afterglow. This was based on the polarization of helium metastable atoms by optical pumping. A chemi-ionization reaction with $\mathrm{CO}_{2}$ produced free polarized electrons. At that time, this source provided results far superior to those of conventional AsGa sources (polarization limited to 50\%). Following a series of improvements the flowing afterglow source could provide a maximum polarization of $87 \%$ at electron currents $I_{\mathrm{e}}=0.1 \mu \mathrm{A}$; but this decreased to $50 \%$ for $I_{\mathrm{e}}=70 \mu \mathrm{A}[2]$.

In 1989 the Institut de Physique Nucléaire at Orsay chose the Rice equipment for the projected European Electron Accelerator Facility (ELFE), but with some changes. The results now obtained at Orsay [3] are very close to those obtained with the Rice source and show the same decrease in polarization at high extracted currents. To explain this effect, we studied sometime ago radiation trapping [4] in the Orsay case [5]; we now extend this discussion to the Rice source. More generally we compare the specific characteristics and the performances of the two sources to propose design improvements.

\section{Comparison Between the Orsay and Rice Sources}

The differences between the two sources $[2,6]$ are shown in Table I. The main one is the choice at Orsay of the electric dipole $\mathrm{D}_{0}$ transition (between the $2 \mathrm{P}_{0}^{3}$ and $2 \mathrm{~S}_{1}^{3}$ substates) for the optical pumping (Fig. 1). The Doppler width at ambient temperature and the splitting between the $2 \mathrm{P}_{1}^{3}$ and $2 \mathrm{P}_{2}^{3}$ states are 1.7 and $2.4 \mathrm{GHz}$, respectively. Thus these transitions are partially 
Table I. - Main differences between Rıce and Orsay sources.

\begin{tabular}{|l|c|c|}
\hline & RICE & ORSAY \\
\hline Gas speed & $\sim 4 \times 10^{3} \mathrm{~cm} \mathrm{~s}^{-1}$ & $1.2 \times 10^{4} \mathrm{~cm} \mathrm{~s}^{-1}$ \\
\hline Optical pumping transition & $\mathrm{D}_{1}$ & $\mathrm{D}_{0}$ \\
\hline Laser & $\begin{array}{c}\text { Single mode } \\
\text { low power (120 } \mathrm{mW})\end{array}$ & $\begin{array}{c}\text { Multumode, } \\
\text { linewidth }=25 \mathrm{GHz} \\
\text { powerful }(1 \mathrm{~W})\end{array}$ \\
\hline Extraction & $\begin{array}{c}\text { Figh vacuum } \\
0200-050 \mathrm{mb}\end{array}$ & $\begin{array}{c}\text { Facuum } \\
005-010 \mathrm{mb}\end{array}$ \\
\hline
\end{tabular}

Rice

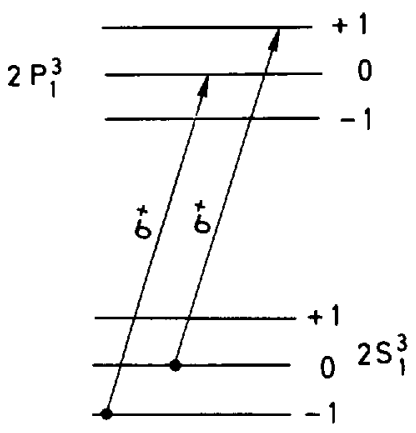

Orsay

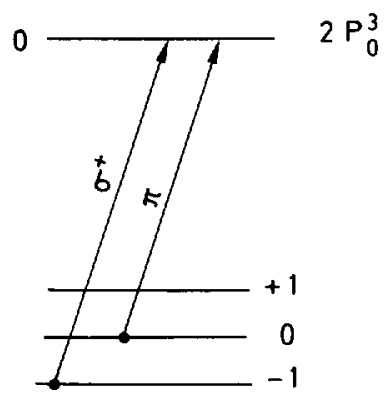

Fig 1. - Optical pumping transition used at Rice and Orsay sources with $\sigma^{+}$and $\pi$ lights.

overlapping. The Rice group built a single mode, low power $(120 \mathrm{~mW})$, stabilized LNA laser [7] and could optically pump on the $\mathrm{D}_{1}$ transition without exciting the $2 \mathrm{P}_{2}^{3}$ state, which can lead to depolarization. Theoretically the $\mathrm{D}_{1}$ transition can give $100 \%$ polarization with a $\sigma_{+}$or $\sigma$ - light beam. At Orsay, optical pumping is undertaken using a commercial Microcontrole Nd-YAG laser equipped with a LNA crystal [8]. This multimode laser has $2.5 \mathrm{GHz}$ linewidth, but is powerful ( 1 Watt) and comparatively inexpensive. Pumping on the $D_{1}$ transition is very difficult or impossible with this laser without exciting the $J=2$ state. However with simultaneous $\sigma$ and $\pi$ pump beams one can pump using the $2 \mathrm{P}_{0}^{3}$ state which is well separated from the $2 \mathrm{P}_{1}^{3}$ state $(\Delta=29 \mathrm{GHz})$. The effective absorption cross-sections, corrected to take, into account the laser width [9], are approximately 6 times higher for the $D_{1}$ transition than for the $\mathrm{D}_{0}$ transition: i.e. the final state density leads to a factor 3 , and an additional factor 2 is due to the laser frequency profile. 
Another difference between the Rice and Orsay systems is the helium bulk flow velocity in the afterglow, which is a factor of two larger in the Orsay source. Thus the metastable decay lengths [10], and consequently the metastable density and the extracted electron current, are larger at Orsay for the same pressure. But metastable atoms in the $2 \mathrm{~S}_{0}^{1}$ state ( $~ 5 \%$ concentration depending on the Laval nozzle tuning) also survive passage down the flowtube (the lifetime is longer than the flight time) and can generate unpolarized electrons. At Rice care was exercised to reduce the background gas pressure in the extraction system. This eliminates electrons due to ionization and excitation of the background gas.

\section{Rate Equation - Radiation Trapping}

The rate equations are different from the Orsay case [5]. If $n^{+}, n^{0}, n^{-}, N^{+}, N^{0}, N^{-}$are the densities of $2 \mathrm{~S}_{1}^{3}$ and $2 \mathrm{P}_{1}^{3}$ atoms, respectively, in the substates $m=+1,0,-1$, at a point $r$ and time $t$, the six equations that describe these densities are:

$$
\begin{gathered}
\frac{\mathrm{d} n^{-}}{\mathrm{d} t}=-\frac{n^{-}}{\tau_{p}}+\frac{N^{-}}{3 \tau}+\frac{N^{0}}{3 \tau}-\frac{n^{-}}{\tau_{\mathbf{r}}} \\
\frac{\mathrm{d} n^{0}}{\mathrm{~d} t}=-\frac{n^{0}}{\tau_{\mathrm{p}}}+\frac{N^{+}}{3 \tau}+\frac{N^{-}}{3 \tau}-\frac{n^{0}}{\tau_{\mathbf{r}}} \\
\frac{\mathrm{d} n^{+}}{\mathrm{d} t}=\frac{N^{+}}{3 \tau}+\frac{N^{0}}{3 \tau}-\frac{n^{+}}{\tau_{\mathrm{r}}} \\
\frac{\mathrm{d} N^{-}}{\mathrm{d} t}=-\frac{N^{-}}{\tau} \\
\frac{\mathrm{d} N^{0}}{\mathrm{~d} t}=\frac{n^{-}}{\tau_{\mathrm{p}}}-\frac{N^{0}}{\tau} \\
\frac{\mathrm{d} N^{+}}{\mathrm{d} t}=\frac{n^{0}}{\tau_{\mathrm{p}}}-\frac{N^{+}}{\tau}
\end{gathered}
$$

where $\tau$ is the lifetime of the $2 P_{1}^{3}$ state $\left(\tau=10^{-7} \mathrm{~s}\right)$ and $\tau_{\mathrm{r}}$ is the relaxation time of metastables. The relaxation time $\tau_{\mathrm{r}}$ describes phenomenologically the loss of metastables to the walls [11] and is calculated $[10,12]$ by $\tau_{\mathrm{r}}=\alpha R^{2} P /(D P)$ with $D P=470$ tor. $\mathrm{cm}^{2} \mathrm{~s}^{-1}$ and $\alpha=0.27$, $R$ and $P$ being the radius and the helium pressure in the source, respectively. A complete discussion of the measurement and calculation of $\tau_{\mathrm{r}}$ at Rice is presented by Keliher [10]. The quantity $\tau_{\mathrm{p}}$ is the so-called pumping time defined by $\frac{1}{\tau_{\mathrm{p}}}=\frac{\sigma_{a} \cdot I_{\mathrm{T}}}{h \nu_{0} \cdot S}$, where $I_{\mathrm{T}}$ is the total laser power distributed over area $S ; h \nu_{0}$ is the energy quantum for a frequency $\nu_{0}=c / \lambda_{0}$, with $\lambda_{0}=1.083 \mu \mathrm{m}$. We denote by $\lambda_{0}$ the wavelength involved in the transition from the metastable "ground state" $2 \mathrm{~S}_{1}^{3}$ to the excited state $2 \mathrm{P}_{1}^{3}$. The level-to-level absorption cross section $\sigma_{a}$ is deduced from the classical state-to-state absorption cross section [9] for unpolarized light

$$
\overline{\sigma_{a}}=\frac{g_{f}}{g_{\imath}} \frac{\lambda^{2}}{8 \pi \tau} F\left(\nu-\nu_{0}\right)
$$

where

$$
F\left(\nu-\nu_{0}\right)=\left[\frac{M c^{2}}{2 \pi k T \nu_{0}^{2}}\right]^{1 / 2} \exp \left[-\left(\frac{\nu-\nu_{0}}{\nu_{0}}\right)^{2} \frac{M c^{2}}{2 k T}\right]
$$


is the Doppler distribution around $\nu_{0}$ [13]; so $\sigma_{a}=\frac{\lambda^{2}}{2 \pi \tau} F\left(\nu-\nu_{0}\right)$. The laser width correction is not justified in the Rice case because its frequency width is negligible. Note the intensity $I=I_{\mathrm{T}} / S$ is the significant parameter rather than $I_{\mathrm{T}}$.

Equations (1) describe the metastable populations in a frame following the gas flow. These populations are a function of time only. The deduced metastable polarization

$$
P o l=\frac{n^{+}-n^{-}}{n^{+}+n^{0}+n^{-}}
$$

is not dependent on $\tau_{\mathbf{r}}$, but only on $\tau_{\mathbf{p}}$. This means that the polarization never reaches a steady state $(t \rightarrow \infty)$, but if the light is intense enough and/or the pumping area great enough, Pol can be large. This description is slightly different for a sealed cell, where a discharge repopulates the metastable sublevels which are destroyed at the cell wall [14]. In this case, repopulation would introduce additional terms $n / 3 \tau_{\mathrm{r}}$ in the rate equations. But calculations using the equation system (1) show that the polarization is not very dependent on these terms. To check the validity of the model, we calculated the polarization as a function of laser power, which was measured at Rice [2] for a low metastable density $\left(n \leq 10^{9} \mathrm{~cm}^{-3}\right)$ for which radiation trapping is negligible. Figure 2 demonstrates that the results agree very well with the experimental data.

Radiation trapping is the reabsorption of unpolarized radiation emitted by the decay of excited states during the optical pumping process. Figure 3 shows all the emission and absorption transitions. To take reabsorption into account, we add to the rate equations (1) specific terms following the procedure proposed by Anderson et al. [15] For example, the additional term to the third equation in (1) has the shape.

$$
-\frac{N^{e}}{\tau} \int \mathrm{d}^{3} \overline{r^{\prime}} \mathrm{d} \nu\left[P_{\Delta m}(\theta) F\left(\nu-\nu_{0}\right)\right] \times \exp \left[-\left(\bar{r}-\overline{r^{\prime}}\right) \sum \sigma_{l}^{k}(\theta)\left(n^{a}-N^{b}\right)\right]
$$

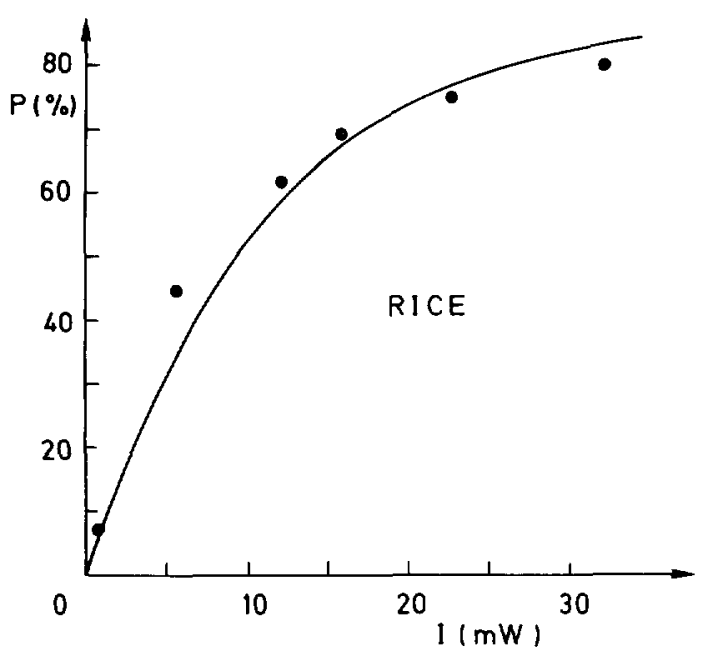

Fig. 2. -- Electron polarization as a function of laser power. Experımental data [2] and polarization calculated without radiation trapping by rate equations (1) The parameters are $I_{\mathrm{T}}=80 \mathrm{~mW}$, $R=5 \mathrm{~cm}$ and $n=10^{9} \mathrm{~cm}^{-3}$ 


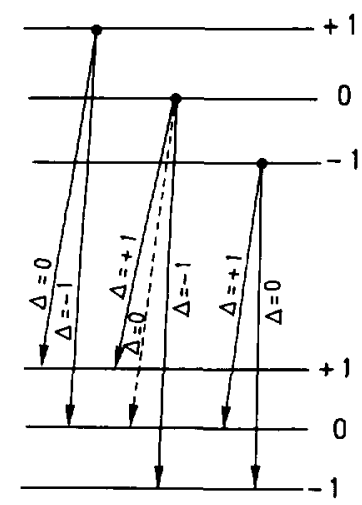

Emission

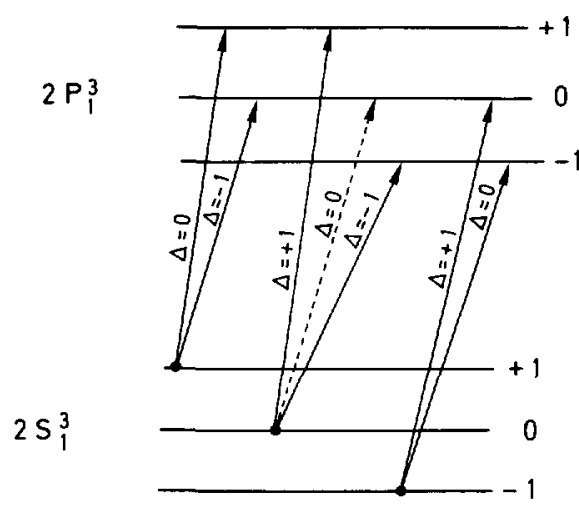

Absorption

Fig. 3 - Emission and absorption transitions involved in electric dipole transition $2 \mathrm{~S}_{1}^{3}-2 \mathrm{P}_{1}^{3}$ for unpolarızed radiations. $\Delta=m_{\mathrm{f}}-m_{1}$ is the magnetic quantum number transfer in these transitions For $\Delta=m_{\mathrm{f}}-m_{\mathbf{t}}=0$ the transition is forbidden.

$$
\times\left[\sum \sigma_{l}^{k}(\theta)\left(n^{0}-N^{d}\right)\right]
$$

where $\theta$ is the angle between $\bar{r}-\overline{r^{\prime}}$ and the direction of the magnetic field.

The first factor is the electric dipole deexcitation probability of the $N^{\mathrm{e}}$ atoms in $2 \mathrm{P}_{1}$ state at the point $\overline{r^{\prime}}$. This radiation is attenuated along the path $\bar{r}-\overline{r^{\prime}}$ by the transition $a \rightarrow b$ (second factor). The last factor gives the radiation absorption by the transition $c \rightarrow d$ at the point $\bar{r}$. The absorption cross section $\sigma_{l}^{k}$ corresponds to the absorption of emitted radiation characterised by the transition $\Delta m=\left(m_{f}-m_{\imath}\right)=k$ and absorbed by the transition $\Delta m=$ $\left(m_{f}-m_{\imath}\right)=l$ These quantities are deduced from the matrix elements of the dipole operator $\bar{D}$ as $|<\imath| \bar{E}_{\alpha}(\theta) \quad \bar{D}|f>|^{2}, \bar{E}_{\alpha}(\theta)$ being the field of the incident wave [7]. Appendix gives the nine values of $\sigma_{l}^{k}(\theta)$ (avallable only for the $\mathrm{D}_{1}$ transition in helium pumping) and the additional term (3) after simplification (same example of the third Eq. (1)).

The NAG code from the CERN library is used to solve the equation system $(1+3)$. The input parameters are:

a) laser power $I_{\mathrm{T}}=80 \mathrm{~mW}$

b) irradiation area $S=40 \mathrm{~cm}^{2}$.

\section{Results and Discussion}

The rate equations give the metastable densities as a function of time. We have calculated the metastable polarization using a flight time corresponding to a length of approximately $6 \mathrm{~cm}$. Note that the experimental uncertainties $(\lambda / 4$ plate, optics, chemi-ionization efficiency, spin singlet $2 S_{0}^{1}$ metastable presence, etc.) are not included although they limit the attainable polarization to below $100 \%$. These corrections are estimated to be approximately $10 \%$ [2]. As the maximum polarization at Rice is $87 \%$, this value is chosen as the normalization factor of the calculated polarization. 
The most serious assumption of the previous calculation (Eq. (4)) was that $n^{2}$ and $N^{3}$ densities are independent of the position $\bar{r}$ whereas they have an approximately parabolic radial profile $[12,17]$. An improvement to the calculations previously presented is to assume that the densities have a $\cos (\pi r / 2 R)$ spatial distribution. This assumption is more realistic because the radiation emitted at $r=R$ has a higher probability to escape than at the center $(r=0)$. Unfortunately, by including density variation, the computer times become unreasonably long. Certainly a more sophisticated procedure must be developed, as suggested by Happer and Redsun [18]. Nevertheless, for a rough understanding of the experimental data of the Rice group the previous calculations are sufficient. In the future we hope to obtain more accurate metastable polarization data and to improve the comparison with sophisticated calculations.

In the present work the cosine function distribution is used only to calculate the polarization values versus the metastable density (Fig. 4) because the results with and without the density correction are very close: at high density $\left(n>10^{10} \mathrm{~cm}^{-3}\right)$ this correction increases the polarization by $10 \%$ approximately. Experimental data previously published [2] are shown on the same figure. The corresponding metastable concentrations were determined by absorption of the light emitted by a helium lamp [10] and are inaccurate. Nevertheless, one sees an agreement despite the crudeness of the reabsorption model. The conclusion is that radiation trapping is responsible for the polarization decrease at high metastable concentrations or high electron currents. Fortunately the Rice results can be improved by changing the experimental set-up and procedure: laser, geometry, etc. Figure 4 also shows the metastable polarization for the Orsay source. In this case the trapping becomes significant only at metastable densities higher than those used at Rice because the effective absorption cross-section is lower for the $D_{0}$ transition than for the $D_{1}$ transition. This proves the value of optical pumping using the $\mathrm{D}_{0}$ transition.

The polarization is calculated as a function of the total laser power (Fig. 5) for two metastable densities $\left(7 \times 10^{9}\right.$ and $\left.10^{10} \mathrm{~cm}^{-3}\right)$. This calculation shows that saturation is almost reached at $I_{\mathrm{T}}=80 \mathrm{~mW}$, as was confirmed by the Rice measurements. Increasing the laser power improves

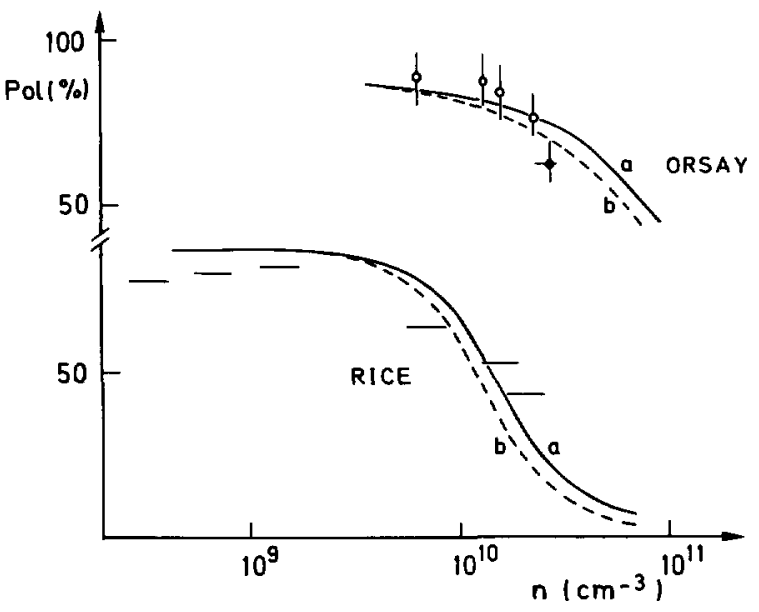

Fig. 4. - Polarization calculated versus the metastable density for the Orsay and Rice sources. The cosine profile is included (a) or not (b). The experimental data are presented with error bars. The empty points correspond to the metastable density measured by laser at Orsay. The other ones (full point and lines) correspond to densities measured by helium light absorption. 


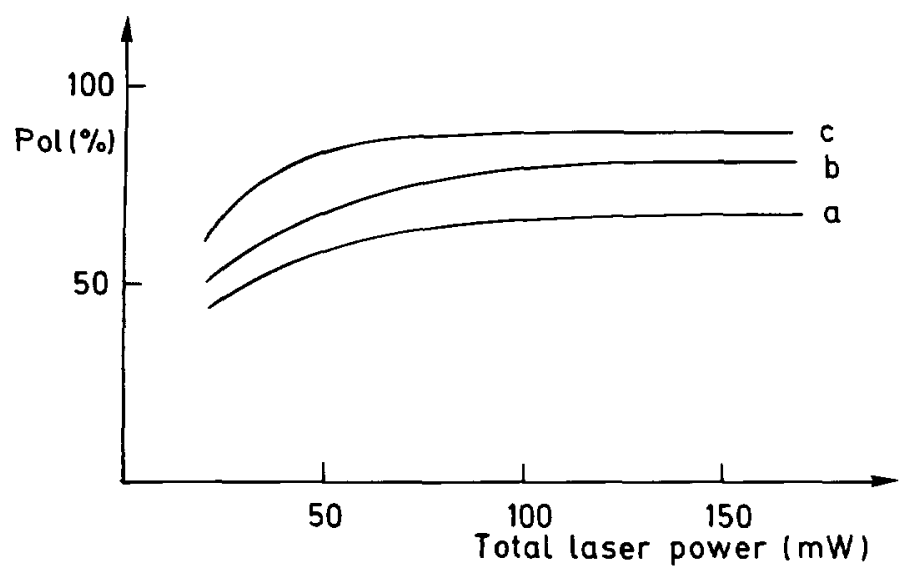

Fig. 5. - Polarization calculated versus the total laser power for metastable density of $10^{10} \mathrm{~cm}^{-3}$ (a) and $7 \times 10^{9} \mathrm{~cm}^{-3}$ (b). The curve (c) is the polarization calculated without radiation trapping correction.

the polarization only slightly. We have assumed that the laser light beam is homogeneous over the pumping volume. In fact, measurements of the light distribution at point $r$ show a Gaussian distribution. This could lead to the unsaturation of some metastables. This effect must be negligible with the Rice set-up, where two baffles concentrate the metastable beam and increase the polarization by $10 \%$ [19]. On the other hand it must play a large role at Orsay. Figure 5 also shows the polarization versus the laser power, calculated without the trapping corrections. Then the polarization is $87 \%$ except at low power ( $I_{\mathrm{T}}$ less than $50 \mathrm{~mW}$ ) when the pumping time $\tau_{\mathbf{p}}$ becomes large.

To reduce the radiation trapping it seems interesting to reduce the dimension of the optical pumping chamber. In this way the metastable density, i.e. the electron current, decreases (the decay length is shorter $[10,12])$. Therefore an optimization process is required.

\section{Conclusion}

From our calculations, it appears that the polarization degradation of the Rice source is mostly due to radiation trapping, as it was suspected by Walters et al. [20,21]. The curves in Figure 4 show clearly that this effect is more important at Rice than at Orsay because the absorption cross-sections are higher for the $D_{1}$ transition: the factor 6 between these cross sections explains approximately the shift between the curves. Since spin polarization is conserved in chemiionization reactions (checked at Rice) and the extraction geometry is identical in the two sources, one expects electron polarization superior at Orsay than at Rice for the same high electron currents. In fact, the experimental values of these polarizations $[2,3]$ are close. This disagreement is presently studied [22].

To increase the polarization at Rice it would be fruitful to use optical pumping by $D_{0}$ transition under the present conditions. After that, some minor improvements in the experimental conditions could be tested to increase the polarization: i) an increase of the laser power per unit area, ii) an improvement of the electron extraction efficiency so as to work at lower metastable densities: in this way, a higher polarization should be reached for the same current, and iij) a reduction of the pumping volume to reduce the trapping probability. Finally, one could use a 
classical technique, i.e. decrease the effective absorption cross sections by detuning the laser frequency to the wings of the atomic resonance line [23].

\section{Acknowledgments}

The author would like to thank G.K. Walters for communicating the Rice experimental data and for many comments and encouragements. He clearly showed the physical differences between the discharge cell and the flowing afterglow and the consequences. Fruitful "intercontinental" discussions with G.W. Rutherford were a great pleasure. Our acknowledgements are due to W. L. Anderson, G. Lampel and B. Cagnac for helpful conversations. We also thank A. Talbot and N. Revoyre for the nice typing of this paper and for their patience.

\section{Appendix}

The same assumptions as those previously proposed by Anderson et al. [15] are used to simplify equations (3):

i) the pumping chamber is a infinitely long cylinder of radius $R(R=5 \mathrm{~cm})$ with nonreflective walls,

ii) the densities $n^{2}$ and $N^{2}$ are taken to be independent of position $\bar{r}$,

iii) the laser light is homogeneous. The integrations over frequency and angle (Eqs. (3)) are performed using Hermitian and Gaussian integratıon procedures. The integration over radius is performed exactly.

The trapping contribution has then the simplest shape $T_{1}+T_{2}+T_{3}$ where:

$$
T_{1}=\frac{\left(N^{0}+N^{-}\right)}{2 \sqrt{\pi} \tau} \sum_{\imath} \omega_{\imath} \sum_{\jmath} \omega_{3 \jmath} F_{3}\left(u_{\jmath}\right)\left(n^{+}-N^{+}\right)+\omega_{1 \jmath} F_{1}\left(u_{\jmath}\right)\left(n^{+}-N^{0}\right)
$$

with

$$
F_{3}\left(u_{\jmath}\right)=2 \sqrt{1-u_{\jmath}^{2}}\left[\frac{1-\exp \left(-\frac{9 \gamma_{2} R \phi\left(u_{\jmath}\right)}{4 u_{\jmath}}\right)}{\phi\left(u_{\jmath}\right)}\right]
$$

and

$$
F_{1}\left(u_{\jmath}\right)=\frac{\left(2-u_{\jmath}^{2}\right)^{2}}{\sqrt{1-u_{\jmath}^{2}}}\left[\frac{1-\exp \left(-\frac{9 \gamma_{2} R \phi\left(u_{2}\right)}{4 u_{\jmath}}\right)}{\phi\left(u_{\jmath}\right)}\right]
$$

and

$$
\begin{gathered}
\phi\left(u_{\jmath}\right)=\frac{u_{\jmath}^{4}}{\left(2-u_{\jmath}^{2}\right)}\left(n^{0}+n^{-}-N^{+}-N^{0}\right)+\left(2-u_{\jmath}^{2}\right)\left(n^{+}+n^{0}-N^{-}\right)+2 \frac{\left(1-u_{\jmath}^{2}\right)}{\left(2-u_{\jmath}^{2}\right)} u_{\jmath}^{2} \\
\times\left(n^{+}+n^{0}+n^{-}-N^{+}-N^{0}-N^{-}\right)
\end{gathered}
$$




$$
\begin{gathered}
T_{2}=\frac{\left(N^{+}+N^{0}+N^{-}\right)}{\sqrt{\pi} \tau} \sum_{2} \omega_{2} \sum_{\jmath} \omega_{5 \jmath}\left(n^{+}-N^{+}\right) F_{5}\left(u_{\jmath}\right)+ \\
+\omega_{3 \jmath}\left(n^{+}-N^{0}\right) F_{3}\left(u_{\jmath}\right)+\omega_{3 \jmath}\left(n^{+}-N^{0}\right) F_{3}\left(u_{\jmath}\right)
\end{gathered}
$$

with

$$
F_{5}\left(u_{3}\right)=\frac{2}{\sqrt{1-u_{3}^{2}}}\left[\frac{1-\exp \left(-\frac{9 \gamma_{2} R \phi\left(u_{\jmath}\right)}{4 u_{3}}\right)}{\phi\left(u_{\jmath}\right)}\right]
$$

and

$$
F_{3}\left(u_{\jmath}\right)=\sqrt{1-u_{\jmath}^{2}}\left[\frac{1-\exp \left(-\frac{9 \gamma_{2} R \phi\left(u_{\jmath}\right)}{4 u_{3}}\right)}{\phi\left(u_{\jmath}\right)}\right]
$$

and

$$
\begin{gathered}
\phi\left(u_{\jmath}\right)=\left(1-u_{\jmath}^{2}\right)\left(n^{+}+2 n^{0}+n^{-}-N^{+}-2 N^{0}-N^{-}\right)+2 u^{2}\left(n^{+}+n^{0}+n^{-}-N^{+}-N^{0}-N^{-}\right) \\
T_{3}=\frac{\left(N^{+}+N^{0}\right)}{2 \sqrt{\pi} \tau} \sum_{\imath} \omega \sum_{\jmath} \omega_{3 \jmath} F_{3}\left(u_{\jmath}\right)\left(n^{+}-N^{+}\right)+\omega_{5 \jmath} F_{5}\left(u_{\jmath}\right)\left(n^{+}-n^{0}\right)
\end{gathered}
$$

with

$$
F_{3}\left(u_{\jmath}\right)=2 \sqrt{1-u_{\jmath}^{2}}\left[\frac{1-\exp \left(-\frac{9 \gamma_{2} R \phi\left(u_{\jmath}\right)}{4 u_{\jmath}}\right)}{\phi\left(u_{\jmath}\right)}\right]
$$

and

$$
F_{5}\left(u_{\jmath}\right)=\frac{1}{\sqrt{1-u_{\jmath}^{2}}}\left[\frac{1-\exp \left(-\frac{9 \gamma_{3} R \phi\left(u_{\jmath}\right)}{4 u_{\jmath}}\right)}{\phi\left(u_{\jmath}\right)}\right]
$$

and

$$
\begin{gathered}
\phi\left(u_{\jmath}\right)=\left(2-u_{\jmath}^{2}\right)\left(n^{0}+n^{-}-N^{+}-N^{0}\right)+\frac{U_{\jmath}^{4}}{\left(2-u_{\jmath}^{2}\right)}\left(n^{+}+n^{0}-N^{0}-N^{-}\right) \\
+\frac{2 u_{\jmath}^{2}\left(1-u_{\jmath}^{2}\right)}{2-u_{\jmath}^{2}}\left(n^{+}+n^{0}+n^{-}-N^{+}-N^{0}-N^{-}\right)
\end{gathered}
$$

$\chi_{2}$ are the nodes and $\omega_{2}$ the weighting factors of the Hermite integration method [24], and $u_{\jmath}$ the nodes and $\omega_{\imath}$ the weighting factors of the Gaussian integration method [24], with:

$$
\gamma_{\imath}=\frac{\lambda^{3}}{8 \pi \tau}\left[\frac{M}{2 \pi k T}\right]^{1 / 2} \mathrm{e}^{-\chi_{2}^{2}}
$$


The cross-sections $\sigma_{\Delta m, \text { absorption }}^{\Delta m, \text { are }}$

$$
\begin{gathered}
\sigma_{0}^{0}=2 \sin ^{2} \theta \cdot \sigma \\
\sigma_{+}^{0}=\sigma_{-}^{0}=\cos ^{2} \theta \cdot \sigma \\
\sigma_{+}^{+}=\sigma_{-}^{-}=\frac{\sin ^{4} \theta}{\left(1+\cos ^{2} \theta\right)} \cdot \sigma \\
\sigma_{0}^{+}=\sigma_{0}^{-}=\frac{2 \cos ^{2} \theta \cdot \sin ^{2} \theta}{\left(1+\cos ^{2} \theta\right)} \cdot \sigma \\
\sigma_{-}^{+}=\sigma_{+}^{-}=\left(1+\cos ^{2} \theta\right) \cdot \sigma \\
\sigma=\frac{3 \lambda^{2}}{32 \pi \tau} F\left(\nu-\nu_{0}\right)
\end{gathered}
$$

\section{References}

[1] Gray L.G. et al., Rev. Scr. Instrum. 54 (1983) 271, and references therein.

[2] Rutherford G.H. et al., Rev. Scı. Instrum. 61 (1990) 1460, and references therein.

[3] Arianer J. et al., Nucl. Instr. Meth. A337 (1993) 1.

[4] Happer W., Rev. Mod. Phys. 44 (1972) 169.

[5] Brissaud I. and Jacquemin C., J. Phys. II France 5 (1995) 93.

[6] Aminoff C.G. et al., Proceedings of the 9th International Symposium, Bonn, September 1990.

[7] Lynn J.G., Ph.D. Thesis, Rice University (1990).

[8] Aminoff C.G. et al., Optrcs Commun. 86 (1991) 92, and references therein.

[9] Mitchell A.C.G. and Zemanski M.W., Resonance Radiation and Excited Atoms (Cambridge University Press, London, 1961).

[10] Keliher P.J., Ph.D. Thesis, Rice University (1974) and references therein.

[11] Phelps A.V., Phys. Rev. 99 (1955) 1307.

[12] Fergusson E.E. et al., Adv. At. Mol. Phys. 5 (1969) 1.

[13] Corney A., Atomic and Laser Spectroscopy (Clarendon Press, Oxford, 1979).

[14] Schearer L.D. and Padetha Tin, Phys. Rev. A 42 (1990) 4028.

[15] Tupa D. and Anderson L.W., Phys. Rev. A 36 (1987) 2142;

Tupa D. et al., Phys. Rev. A 33 (1986) 1045.

[16] Cagnac B., private communication.

[17] Bolden R.C. et al., J. Phys B3 (1970) 45.

[18] Happer W., private communication.

[19] Ratliff J.M., Ph.D. Thesis, Rice University (1989).

[20] Keliher P.J. et al., Phys. Rev. A 1 (1975) 1279.

[21] Walters G.K., talk given at Université d'Orsay (1989) and private communication.

[22] Brissaud I., Nucl. Instr. Meth. A354 (1995) 588.

[23] Redsun S.G., Ph.D. Thesis, Prınceton University (1990).

[24] Abramowitz M. and Stegun I.A., Handbook of Mathematical Functions with Formulas, Graphs and Mathematical Tables (Dover, New York, 1970). 